\title{
Ueber die verschiedenen Systeme von Nervenfasern im Balken des Menschen.
}

\author{
Von \\ Prof. Dr. G. MINGAZZINI \\ in Rom. \\ (Hierzu Taf. XI-XII.)
}

Es ist das Verdienst von Marchiafava und Bignami im Corpus callosum der Alkoholisten wie auch in der Commissura anterior eine Degeneration entdeckt $\mathrm{zu}$ haben ${ }^{1,2,3,4}$ ), welche immer die mittleren Schichten des Balkens ergreift.

Sie haben folgende Tatsachen festgestellt:

1. Die Degeneration betrifft die mittlere Schicht des Balkens in bald größerer. bald geringerer Ausdehnung (von oben nach unten), während die dorsale und ventrale Schicht stets freibleiben:

2. die Fasern der Striae Lancisi bleiben stets verschont;

3. die Fasern des hinteren Teiles des Balkens (Splenium) bleiben sehr häufig von dem degenerativen Prozesse verschont.

Die Untersuchungen dieser Forscher haben mich veranlabt. die meinigen zum Vergleich heranzuziehen, die ich vor ungefähr zwölf Jahren über die Markscheidenbildung im Balken von menschlichen Föten und Neugeborenen angestellt habe ${ }^{5}$ ). Diese im allgemeinen wenig bekannten Lntersuchungen waren darauf gerichtet, den Zeitpunkt festzustellen, in dem die Markscheiden im Balken (und in den benachbarten Teilen. Fornix, Septum pelluc. etc.) auftreten, und können nach meiner Meinung uns verständlich machen, warum der Degenerationsprozeß einen anatomisch gut umschriebenen Sitz hat. Indem ich die Palsche Methode anwendete und Serienschnitte des Balkens von menschlichen Föten und Neugeborenen von vorn nach hinten untersuchte, gelang es mir, die Periode des Auftretens von Markscheiden in den Balkenfasern in drei Stadien einzuteilen:

1) Marchiafara und Bignami. Sopra un' alterazione del corpo calloso etc. Riv. di patol. nerv. Firenze 1903.

2) Bignami, Sulle alterazioni del corpo calloso ete, Policlinico, Sez. prat. 1907 . p. 460.

$\left.{ }^{3}\right)$ Marchiafara und Bignami. Sopra un' alterazione sist. etc. Rendic. della R. Accad. dei Lincei. Sed. del. 6. Febr. 1910.

4) Marchiafava, Bignami und Nazari, (Monatsschr. f. Psych. Bd. 29. 1911.) Vgl. auch O. Rossi. Sull' istiol. patol. di una speciale alter. Riv. di pat. nerv. p. 346.1910.

3) Mingazzini. G.. Osservaz. anat. intorno al corpo calloso etc. (Ric. fatte nel Labor. di Anat. um. di Roma. Vol. VI. $1897-98$ p. 5 e sers.)

Monatsschrift f. Psychiatrie u. Neurologie. Bd. XXxI. Heft $6 . \quad 35$ 
Im ersten Stadium, das von der 2. bis 3. Woche des extrauterinen Lebens bis ungefähr zum Ende des 2. Monats reicht, zeigen die Balkenfasern beginnende Markscheidenbildung entsprechend den dorsalen und ventralen Randpartien (Fig. 3). Ich benenne diese beiden Streifen von markumhïllten Fasern mit dem Namen Laminae marginales (dorsalis und ventralis).

In den Längsschnitten des Balken eines viermonatlichen Kindes (Fig. 2) sieht man Fasern, welche in antero-posteriorem (sagittalem) und in frontalem Sinne gelagert sind, was beweist, daß die Fasern des Corpus callosum nicht nur in parallelen (frontalen), sondern auch in schrägen und vertikalen Ebenen verlaufen. Dies bestätigt noch einmal die Angabe der Mehrzahl der Anatomen (Ganser. Dejerine, Kölliker), daß die Balkenfasern in den verschiedensten Richtumgen verlaufen.

Im zweiten Stadium, das vom dritten resp. vierten bis zum siebzehnten Monat reicht, dehnt sich die Markscheidenbildung nicht nur auf die seitlichen und medianen Teile des Balkens. sondern auch tiefer gegen die mittlere Zone desselben aus. Diese Markscheidenbildung ist jedoch um so schwächer, je mehr man sich der Mittellinie nähert, wo die Fasern fast marklos erscheinen (Fig. 4). Wenn man die Balkenfasern in drei Schichten einteilt, eine oberflächliche, eine tiefe und eine mittlere, so bemerkt man, daß die Fasern der letzteren Schicht zum Teil bereits von Markscheiden umgeben sind, aber stets in geringerem Maße als die beiden Randschichten. Die beiden neuen Streifen von markumhülten Fasern, deren jeder sich dem betreffenden Randstreifen angelagert hat, verdienen den Namen Laminae profundae.

In einem weiteren Stadium (vom 17. Monat ab) ergreift die Markscheidenbildung noch mehr das Gebiet der Laminae profundae und auch die Mittelschicht, welche ich nach dem Vorschlage Marchiafavas als Lamina media bezeichne (Fig. 5). Doch bleibt ein kleiner, dieser letzten Lamina entsprechender Streifen noch frei von markhaltigen Nervenfasern.

Vergleicht man nun diese dem Studium der Ontogenese des menschlichen Gehirnes entnommenen Ergebnisse mit denjenigen, die sich aus dem Studium der Degeneration der Balkenfasern bei Alkoholisten ergeben, so scheint es mir keinem Zweifel zu unterliegen, daß im Balken wenigstens drei Systeme von Nervenfasern vorhanden sind. Die Fig. 7, welche der Arbeit von Marchiafara und Bignami (4) entnommen ist, bezieht sich auf einen Fall, in dem die Degeneration ausschließlich (und zwar vertikal in sehr beschränkter Ausdehnung) die mittlere Schicht des Balkens (Lamina media) betrifft. Diese Figur entspricht genau derjenigen (Fig. 5), welche sich auf das Corpus callosum eines Kindes zwischen dem $4 \frac{1}{2}$. und 17 . Monat bezieht. Die Balkenfasern sind hier größtenteils vollkommen mit Markscheiden umhüllt, abgesehen von den Fasern, die in der Mittelschicht verlaufen. Die Mittelschicht des Balkens, welche beim menschlichen Fötus sich zuletzt mit Markscheiden umgibt, entspricht also derjenigen, welche zuerst bei Alkoholisten degeneriert. 
Die Figuren s und 9, welche der Arbeit (4) von Marchiajara und Bignami entnommen sind, zeigen einen anderen lall, in dem nicht allein die mittlere Sehicht (Lamina media), sondernauch die Laminae profundac (dorsalis und ventralis) degeneriert waren. Diese begeneration läßt also nur die Laminae marginales frei. Siecntspricht jener Periode des extrauterinen Lebens, in weleher nur die Laminat marginales (d. h. die obroflächlichen Schichten) des Balkens. Markscheiden besit\%en. wie man in Fig. 3 sieht, die den Balken fines Kindes ron $1 \frac{1}{2}$ Monaten darstellt. Daraus ist es gestattet. den Schlub \%u ziehen, dab es im Balken drei Gattungen von Fasern gibt (Laminae superficiales, Laminae profundae, Lamina media). deren jede sich zu versehiedenen Zeiten mit Markscheiden mhiullt und dementsprechend isoliert oder gleichzeitig mit den anderen degenerieren kann, wie es bei jedem anderen Faserbündel des Zerebrospinalsystems vorkommt. Sic haben wahrseheinlieh eiren besonderen Ursprung. cinen verschiedenen Verlauf and verschiedene physiologische Bedeutung.

Marchiafard und Bignami machen zwat die Bemerliung. dabs das spätere. Iuftreten von Markseheiden in der Jamina media im Vergleich mit der ventraten und dorsalen Schicht nicht die Bedeutung habo, da B die erstere ein anderes System darstelle als die beiden letzteren: aber in Wirklichkeit bringen sie keinen Beweis für ihre Behauptung bei, was $1 \mathrm{~m}$ so wänschenswerter gewesen wäre, als bis jet\%t alle Forscher seit Flechsig dieses Prinzip an. genommen haben. Vielleicht spiclen sie auf den bereits von Dejerine erhobenen Einwand an, dals nämlich ein System von sehr langen Novenfasern, wie z. B. das System der Pyranidenbahnen. sich nach und nach von oben nach unten mit darkscheiden magehen kamu. also nicht gleichzeitig in seinem ganzen Verlauf. Daher ergibt sich nach Dejerine die Möglichkeit, dals man die Markumscheidung eines Fasersystemes zu cinem Zeitpunkte beobachtet. wo der proximale Anteil des Svstemes bereits mit Markscheiden umgeben ist, der distale aber nicht. Dieser Lmstand kömnte nach Dejerine irreführen und die Vermutung anflommen lassen. dab es sich um zwei versehiedene Systeme handle. Dieser Einwand läßt sich jedoch nicht auf das Auftreten von Markscheiden in den Fasern des Balkens anwenden: er hätte wohl eine Berechtigung, wenn es sich darum gehandelt hätte. dic Beziehungen festzustellen \%. B. zwischen bestimmten Zügen von Nervenfasern des Centrum ovale (oder der Marksubstanz der Hirnwindungen) und den Balkenfasern. Nimmt man an. da B, das Auftreten von Markseheiden von der Hirminde gegen den Balken zut fortschreitet, so ist es klar, dal3 der Befund von Markscheiden in einer Schicht von Nervenfasern der Marksubstan\% des Großhirns zu einem Zeitpunkte des kindlichen Lebens. wo sic im Balken noch nicht vorhanden sind, keinen sicheren Beweis dafür liefern würde, daß die beiden Fasersysteme von einander verschieden sind. Aber bei der Myelinumhällums der verschiedenen Balkenfasern handelt es sich um ein System ron Fasern. Welche 
parallel oder fast parallel übereinander in transversaler Richtung verlaufen, und nicht um ein Fasernsystem, das wie im oben zitierten Beispiel der Pyramidenbahn, von der Hirnrinde ausstrahlend, nach abwärts in die tiefer darunterliegenden Schichten steigt. Deshalb zwingt die Feststellung einer Periode des kindlichen Lebens, in der die beiden Balkenrandschichten eine vollständige Entwickelung der Markscheiden zeigen, während der Rest des Balkens (die Laminae profundae und die Lamina media) myelinfrei ist, zu der Annahme, daß man es hier mit ganz gesonderten Systemen von Nervenfasern zu tun habe.

Was die jetzt sogenannte Lamina media, d. h. diejenigen Fasern, welche sich in der letzten Periode mit Markscheiden umgeben, anbelangt, so blieb ich tatsächlich unsicher, ob sie ein (drittes) System von Fasern bilden, das verschieden ist von denjenigen, welche sich in der zweiten Periode mit Markscheiden umgeben. Ich habe in meiner Arbeit daran erinnert, daß die Balkenfasern - nach den Untersuchungen von Ramon y Cajal - die Collateralen oder die Teilungsäste der langen Assoziationsfasern darstellen. Wenn nun, wie es natürlich ist, das Auftreten von Markscheiden in den Balkenfasern vom Ursprungsort des Neurons gegen dessen peripheres Ende zu fortschreitet, so hatte ich darau. geschlossen, daß das teilweise Fehlen von Markscheiden in den Balkenfasern (d. i. in denen, welche speziell in den mittleren Schichten gelegen sind) in einer bestimmten Periode des extrauterinen Lebens vielleicht von dem Umstande abhängen könnte. daß die Markscheidenbildung bis zum siebzehnten Monate in der mittleren Schicht sich nur bis zur homolateralen Hälfte des Balkens erstreckt hatte, und daß sie sich also späterhin auch auf die kontralaterale Hälfte desselben erstrecken werde. Aus diesem Grunde war ich im Zweifel geblieben, ob die Fasern der genannten Lamina wirklich ein spezielles System darstellen. Jetzt beseitigt das Studium der von der menschlichen Pathologie ermittelten Degeneration des Balkens diese Zweifel. Sie zeigt uns vor allem, daß die Lamina media isoliert degeneriert, und daß daher die zuletzt sich mit Markscheiden umgebenden Balkenfasern in der Tat ein besonderes System darstellen.

Wenn man überdies erwägt — was auch aus der Fig. 5 ersichtlich ist - , daß die Markscheidenbildung in dem der Lamina media entsprechenden kleinen Balkenstreifen auch im 17. Monate noch nicht ganz vollendet ist, so kann man berechtigterweise den Schluß ziehen, daß das zweite, den Laminae profundae entsprechende Fasernsystem (also dasjenige, welches sich in dem langen Zeitraume zwischen dem vierten und dem siebzehnten Monate mit Markscheiden umhüllt) lange Zeit braucht, um sich mit Myelin zu umkleiden. Dies stimmt übrigens mit der Tatsache überein, daß es den größten Teil der Balkenfasern umfaßt.

Aus den Ergebnissen dieser Studien ist also der Schluß gestattet, daß die Balkenfasern aus drei Systemen bestehen, die sich in verschiedenen Zeitperioden mit Markscheiden umgeben. In 
einer ersten Periode, welche die drei ersten Monate des intrauterinen Lebens umfaßt, umgeben sich die Randpartien des Balkens mit Markscheiden; damn — zwischen dem vierten und siebzehnten Monate - die darunterliegenden Schichten (Laminae profundae), und in der letzten, vom 17. Monate angefangen, die Lamina media. Es sei bei dieser Gelegenheit daran erinnert, daß im allgemeinen dis Neurologen in der Annahme übereinstimmen, daß diejenigen Fasersysteme, welche sich in der Ontogenese zuletzt mit Markscheiden umgeben, auch in der Phylogenese die jüngsten sind. Da num hiernach die Lamina media eine neophyletische Bildung darstel!t. so begreift man, weshalb sie dem toxischen Prozesse wenig Widerstand leistet und weshalb die Laminae profundae demselben erst später anheimfallen, während die Laminae marginales, welche die. Bedeutung einer palaeophyletischen Formation haben, am widerstandsfähigsten sein müssen.

Dieser Punkt muß besonders betone werden, weil nach der Meinung von Marchiafava und Bignami die große Variabilität in der Ausdehnung der degenerierten Partie von dem Umstande abhängt, daß die degenerierte Schicht infolge eines langsamen atrophischen Prozesses mit der Zeit allmählich an Ausdehnung verliert. Sie nehmen an, daß die Degeneration in den verschiedenen Fällen immer ungefähn die gleiche Inzahl yon Balkenbündeln ergreift und daß daher immer die gleiche Anzahl von (oberflächlichen) dorsalen und rentralen Fasern verschont bleibt. Nun genügt es die beiden Figuren 7 und \& miteinander zu vergleichen. um sich davon zu überzeugen, daß mabhängig von der Ausdehnung der degenerierten Zone die Zahl der verschonten Fasern in der Fig. bei weitem größer ist als in dem Falle, den Fig. S darstellt; und da B in diesem letzten Falle das Gebiet des Corpus callosum sehr stark geschrumpft sein müßte, was man unmöglich zugeben kann.

Der zweite Punkt, den ich betonen will. ist, daß die Strine Lancisii bei den alkoholischen Degenerationen des Balkens stets intakt gefunden worden sind. Um dies verständlich zu machen. sei daran erinnert, daß ich bereits (in meiner oben zitierten Albeit) mit dem Namen Stratum Lancisianum (Fibrae perforantes Köllikers) jenes deutlich umsehriebene Nervenbündel (Fig. (6) bezeichnet habe, dessen Fasern in vertikaler Richtung innerhalb der Striat. Lancisii verlaufen, um es ron andern in sagittaler Richtung verlaufenden Fasern zu unterseheiden. Diese Benemung ist notwendig, weil in den StriaeLaneisii auch Fasern in sagittaler Richtung verlaufen. Um die Bedentung dieser beiden Arten von Fasern verständlich zu machen, muß man daran erinnern, daß sowohl die Treniae tectae als auch die Nervi (Striae) Lancisii dic Repräsentanten einer Windung (Indusetm griseum) sind, welche beim Menschen in Rückbildung begriffen ist. Diese Windung besteht aus zwei weißen Schichten, einer oberflächlichen und einer tiefen. Die erstere wird von einer Lage tangential verlaufender Fasern von verschiedener Dieke, je nach dem Individuum und der Schnittebene. gebildet und verläuft, nach Charpy in der Substantia reticularis 
Arnoldi occipitalwärts. Die tiefe Schicht besteht nach Charp!y ebenfalls aus Fasern von sagittaler Verlaufsrichtung, welche sich frontalwärts in den Pedunculus corporis callosi, occipitalwärts in die weiße Substanz des Gyr. hippocampi fortsetzen. Obersteiner und mit ihm viele andere nehmen ausdrüeklich an, daß diese Schicht das Mark des Induseum griseum darstellt. Ich habe nun nichts Bestimmtes bezüglich des Zeitpunktes feststellen können, in dem dic tangentialen Lancisischen Fasern sich mit Markscheiden umgeben. weil es mir nicht gelungen ist, sie in den Längsschnitten zu bcobachten, welche ich bei dem Gehirn eines viermonatlichen Kinds (Fig. 1) angefertigt habe. Was die Fasern des Stratum Lancisianum anbelangt, so geht aus meinen Untersuchungen an Kindergehirnen von verschiedenem Lebensalter hervor, daß sie genau in der dritten Woche des extrauterinen Lebens entsprechend der Basis der Striat (Fig. 3) sich mit Markscheiden zu umhüllen beginnen, und da 3 von hier aus die Markscheidenumhüllung allmählich nach oben fortschreitet, bis sie etwa im sechsten Monat die Peripherie der Striae erreicht (Fig. 6). Es ist sehr bemerkenswert, daß sowohl das Stratum Lancisianum (d. h. die Fibrae perforantes) als auch die auf der Basider Taenia tecta (Fig. 3) gelegene Schicht sich zu einer Zeit mit Markscheiden zu umhüllen beginnen (in den ersten drei Wochen des extrauterinen Lebens), wo die Balkenfasern noch fast gan\% marklos sind. Hieraus folgerte ich, daß diese Schichten ein phylogenetisch altes System darstellen, das von den anderen transversal verlaufenden Fasern des Balkens unabhängig ist. Dies erklärt ihre Widerstandsfähigkeit gegen den systematischen Degenerationsprozeß des Balkens. Marchiafava und Bignami fanden in der Tat sogar in denjenigen Fällen, in denen die Degeneration der Balkenfasern schwer und vorgeschritten war, die Fasern der Striat Lancisii frei.

Noch in einem anderen Punkte stimmen meine embryologischen Untersuchungen des Balkens mit den Ergebnissen de: Studiums der Degeneration des Balkens bei Alkoholisten überein, nämlich in dem Verhalten der Fasern des Splenium. Flechsig ${ }^{1}$ ) hatte bereits erwähnt, daß die Fasern des Splenium bei Kindern von wenigen Wochen sich mit Markscheiden umgeben, und zwar früher als die anderen Assoziationssysteme, besonders Faserbündel, die, wie er meint, der Sehsphäre angehören. Ich konnte diese Annahme weder bestätigen noch ihr widersprechen, da ich nicht weiß, mittels welcher Kriterien es Flechsig gelungen war, diese zwei Arten von Fasern im Splenium zu unterscheiden. Aber ich möchte die von mir beobachtete Tatsache hervorheben, daß die Markscheidenumhüllung im hinteren Drittel des Balkens sich später vollzieht als in den zwei vorderen Dritteln desselben. Diese Ergebnisse sind durch weitere Studien ergänzt worden. welche das Vorhandensein von zwei verschiedenen Arten von Fasern im Balken nachweisen. In der Tat kann man nach Dejerine $\left.{ }^{2}\right)$

$\left.{ }^{1}\right)$ Flechsig, Gehirn und Seele. Leipzig 1896. Fig. 3.

$\left.{ }^{2}\right)$ Dejerine, Anat. des centres nerveux. Paris, pag. 791. T. I. 
im Splenium des Balkens drei Teile unterscheiden: Einen oberen (hinteres Ende des Truncus c. callosi), einen mittleren (das eigentliche Genu posterius corp. callosi) und einen umgebogenen (Splenium sensu strictiori). Während nun die Strahlungen des oberen Teiles sich wie jene des Truncus corp. callosi verhalten, haben der mittlere und der umgebogene (oder untere) Teil einen verschiedenen Trsprung und verschiedene Bestimmung. Die letzten stammen von der Okzipitalrinde und vom hinteren Teile des Lobus parietalis. umgeben in Gestalt eines vollständigen Ringes das Hinterhorn und bilden zwei Bündel, von denen das obere den Forceps major, das untere den Foreeps minor darstellt; Anders hingegen verhalten sich die Strahlungen des oberen Teiles des Spleniums sowie die rom Truncus corp. callosi stammenden Fasern. Von $\mathrm{P}_{1}$ und $\mathrm{P}_{2}$, vom Praccunus und rom hintere? Teile des G. corp. callosi stammend, durchqueren sie die Assoziationsfasern, dieProjektionsfasern und die Faserndesokeipitofrontalen Bündels, um sich zum Splenium zu begeben. Das Vorhandensein von zwei Systemen von Nervenfasern im Splenium, (in sensu lato) von denen das eine, den größeren Teil umfassende einen anderen Ursprung und eine andere Bestimmung hat als der Rest de: Balkenfasern, erklärt die Tatsache, daß sich die Bildung von Markscheiden dort später vollzieht als hier. Jetzt haben wir eine Gegenprobe hierfür in der Balkendegeneration der . Ilkoholisten, da Marchiafara und Bignami fanden, daß die Fasern des Splenium oft unversehrt bleiben. Es ist nicht unwahrscheinlich, daß eben die Forcepsfasern (wahrscheinlich die Sehfasern) in diesem Teile des Balkens vorherrschen und daher dem Krankheitsprozeß mehr Widerstand leisten als die spärlichen Kommissurenfasern.

\section{Erklärung der Abbildungen auf Tafel XI-XII.}

Allen Figuren gemeinsame Bezeichnungen:

Is d. Lamina superficialis dorsalis (des Balkens); $1 \mathrm{~s} \mathrm{v}$, Lamina superficialis ventralis; $1 \mathrm{p}$, Lamina profunda; $1 \mathrm{~m}$, Lamina media, $\mathrm{s} 1$ Stratum lancisianum (Fibrae perforantes); d, Margo dorsalis corp. callosi: v, Margo ventralis corp. callosi. e v f, Columna fornicis; c c, Corpus callosum. Alle Schnitte der Figuren 1-i waren nach der Palschen Methode (Hämatoxylin) gefürbt.

Fig. 1. Sagittalschnitt durch die hintere Hälfte des Balkens (lateroler Teil eines viermonatlichen Kindes (Reichert, Oc, 3, Objekt. 3).

(Der Schnitt ist im Bereiche einer Stria (st) Lancisii geführt). f $1 \mathrm{t}$, in mehreren Schichten gelagerte Tangentialfasern des Stratum Lancisianum; $\mathrm{s} b$, andere Fasem dieses Stratums.

Fig. 2. Sagittalschnitt der vorderen Hälfte des Bälkens (medialer Teil) von demselben Kinde wie Fig. 1. g, Genu corporis callosi.

Man sieht entlang dem Balken sehr spärliche mit Markscheiden umgebene Fasern von antero-posteriorem Verlaufe, gemischt mit anderen zahlreicheren (Fibrae perforantes Köllikers) von vertikalem Verlaufe, die von den Striae Lancisii kommen. f 01 , entsprechend der ventralen Oberfläche des Balkens verlaufende Fasem. die ich als Fornix longus gedeutet habe.

Fig. 3. Frontalschnitt durch den hinteren Teil des Balkens eines $1 \frac{1}{2}$ Monate alten Kindes.

Die Fasern des Balkenkörpers zeigen beginnende Markscheidenbildung. Die (oberflächliche) Fasern des dorsalen Teiles des Balkens (Lam. suporf. 
dors.) sind spärlich mit Markscheiden umhüllt, viel besser dagegen diejenign der Lamina ventralis, die sich als Fornix longus in die Crura posterior: fornicis erstrecken. Jer basale Teil der das Stratum Lancisianum bildenden Fisern ist intensiv schwarz gefärbt, ebenso die Schicht von longitudinalen Fasern der Lamina dorsalis (ls d), welche (lf i) unter dier Tienia tecta (lateralis) gelegen ist, dio inmitten des überbleibenden Balkenfeldes fast frei von markhaltigen Fasern hervorspringt.

Fig. 4. Frontalschnitt entsprechend der vorderen Hälfte des J3alken. eines $4 \frac{1}{2}$ Monate alten Kindes.

Die Fasern der lateralen Teile des Balkens sind nicht vollständig mit Markscheiden umgeben. Die Markscheidenbildung nimmt gegen die Medianlinie zu ab, namentlich links, wo man die der Lamina media entsprechend. Schicht frei von markhaltigen Fasern sieht. Das Stratum Lancisianum enthält dicke, vertikale, markhaltige Fasern (Fibrae perforantes Köllikers); spärlichere Fasern erscheinen im zentralen Teile der Striae. Vollständig ist dre Markscheidenbildung in dem Faserbündel, welches die mediale Höhlengrenzschicht und die laterale Schicht (s 1) der Lamina septi pellucidi bildet. Man sieht, daß sich die Fasern des Fornix longus scheinbar mit jenen der medialen Grenzschicht des Sept. pelluc. verbinden.

Fig. 5. Frontalschnitt durch den Balken (entsprechend seiner vorderen Hälfte) eines Kindes von 17 Monaten.

Die den Laminae superf. und prof. entsprechenden Fasern des Balken. sind fast vollständig mit Markscheiden umgeben, nur sind die in der Mitt. desselben (Lamina media) verlaufenden ein wenig blass. Die Crura anteriora iornicis zeigen allenthalben gut ausgebildete Markscheiden. aber im Zentrum sind sie etwas blaß. Vom Zentrum des Balken. sieht man sich Fasern (Fornix longus) loslösen, welche fast vertikal verlaufend sich in die Crura anteriora fornicis begeben. Im G. cinguli sicht man viele strahlentörmige Fasern mit Markscheiden; die transversalen Fasern in demselben sind sehr spärlich. (Der linke Teil des Balkens ist nur in seinen Konturen gezeichnet.)

Fig. 6. Dorsaler 'Teil eines Frontalschnittes durch den vorderen 'Teil des Balkens entsprechend einer Stria Lancisii. Das Gehirn stamme von einem $6^{1 / 2}$ Monate alten Kinde.

Die vertikalen Fasern (Fibrae perforantes) des Stratum Lancisiantut haben gut ausgebildete Markscheiden, reichen bis zur Peripherie der Stria Lancisil und ciringen in der Tiefe zwischen die am weitesten dorsalwärts gelegenen transversalen Fasern des Corpus callosum ein. Die die dorsale Schicht (der Lam. suporficial.) des Balkenkörpers bildenden Fasern sind fast vollständig mit Markscheiden umgeben.

Fig. 7. Vertiko-transversaler Schnitt durch das Corpus callosm in «ler Höhe des Koptes des Nucleus caudatus von einem Falle beginnender und wenig ausgebreiteter Degeneration des Corpus callosum. (Nach Marchiafavc und Bignami.) Färbung nach Weigert-Pal.

Die zentrale (mittlere) Zone ist sehr blak und degeneriort und wird in den medialen Partien des Balkens noch schwerer unterscheidbar, insbesondere in der Medianlinie. Seitwärts breitet sie sich in Keilform aus.

Fig. 8. Vertico-transversaler Schnitt durch das Corpus callosum (eines Alkoholikers) entsprechend dessen vorderem Drittel in einem Falle sehr ausgedehnter Degeneration (nach Marchiajara und Bignami).

Die sehr ausgoclehnt (blasse) degenerierte Zone umfaßt fast zwei Drittel des Balkens. Die oberflächlichen Markstreifchen sind erhalten; sic hiben keine scharfen Grenzen, sondern verlieren sich allmählich. Seitwärtwird die degenerierte Zone mächtiger, enthält aber auch zahlreiche gut erhaltene Markfasern. Marchische Methode.

Fig. 9. Vertiko-transversaler Schnitt durch das Corpus callosum (entsprechend seinem vorderen Drittel) eines Alkoholikers. Nach Marchiafaic und Bignami (Färbung Weigert-Pal.)

Man sieht eine sehr ausgebreitete Degeneration des Balkens, dio nur die oberflächlichen Markfasern frei läßt. Immerbin ist die Degeneration in vertiko-transversalen Sinne weniger ausgebreitet als im vorhergehenden. Schnitte. 